\title{
Functions and Future Applications of F1 ATPase as Nanobioengine -
}

\section{Powering the Nanoworld!}

\author{
Sandip S. Magdum* \\ Amity Institute of Biotechnology, Amity University, Noida 201303, India \\ *Email: sandipmagdum@gmx.com
}

Keywords: Nanotechnology, Nanobiomolecules, Nanomechanical, F1 ATPase, Nanobioengine

\begin{abstract}
Recent nanotechnological revolution mandates astonishing imagination about future nanoworld. Nature has ability to create nanobiomolecules which can function in extraordinary way which can be used to produce nano hybrid systems. The opportunity to use such nanobiomolecules in combination of nanomechanical systems for development of novel nano hybrid systems for their various applications needs to explore in further nanotechnological development. F1 ATPase is a subunit of ATP synthase, which is one of the biomolecular structure works on the plasma membrane of the living cell. The reversible function of F1 ATPase gives a counterclockwise rotation of $\gamma$ shaft by hydrolyzing ATP and the energy released in the form of rotational torque. This rotational torque of F1 ATPase can be used to power the functional movement of nanodevice. This feature article discusses comparisons of various biomolecular motors for their powering capacities, recent developments, presents new discoveries, experimentations on F1 ATPase and its novel imaginary futuristic applications where F1 ATPase could be used as nanobioengine for powering functional nanoworld.
\end{abstract}




\section{Introduction}

Nanotechnology is a field of applied science focused on the design, synthesis, characterization and application of materials and devices on the nanoscale [1]. An individual biomolecule seems to be lifeless, but extraordinary physiochemical and functional combination of these biomolecules composes in living things. Living cells acquires biomolecules covering a wide range of molecular dimensions from $0.5 \mathrm{~nm}$ (alanine) to nearly 20,000 $\mathrm{nm}$ (living cell). Regardless of the enormous diversity in form and role, cells and organisms share a common biochemistry. Components of living cells are isolated by the boundaries of membranes and they give responses and communicate to the world by controlling physiochemical actions and reactions. Some proteins have roles to act by structural changes for stabilization of free energies dependent on the local environment. Noncovalent interactions influences on structural and functional changes of proteins, which are hydrogen bonds, hydrophobic interactions, electrostatic bonds, and van der Waals forces, are responsible for conformational changes. Out of various biomolecular motors which is discussed in this review, ATP synthase showing very interesting structural and functional properties suitable as ideal torque generating molecular motor suitable to produce nanohybrids. Kinosita's single molecule physiology lab, a Japanese group working on ATP synthase complex from the last two decades for unraveling knowledge and functions suitable to use in nanobiotechnological applications. F1 ATPase related experimental explorations started from direct observation of the rotation of gamma shaft, its rotational efficiency, synthesis of hybrid nano device, controlling rotation of gamma shaft, mutational improvement of F1 ATPase were carried out $[2,3,4]$. Nanobiomolecular research journey and novel findings of biomolecular capability for their ideal functions is continuing their efforts shown in Fig.1. These ideally functioning biomolecular entities always stressing us to find a suitable place for their efficient use in various applications. 


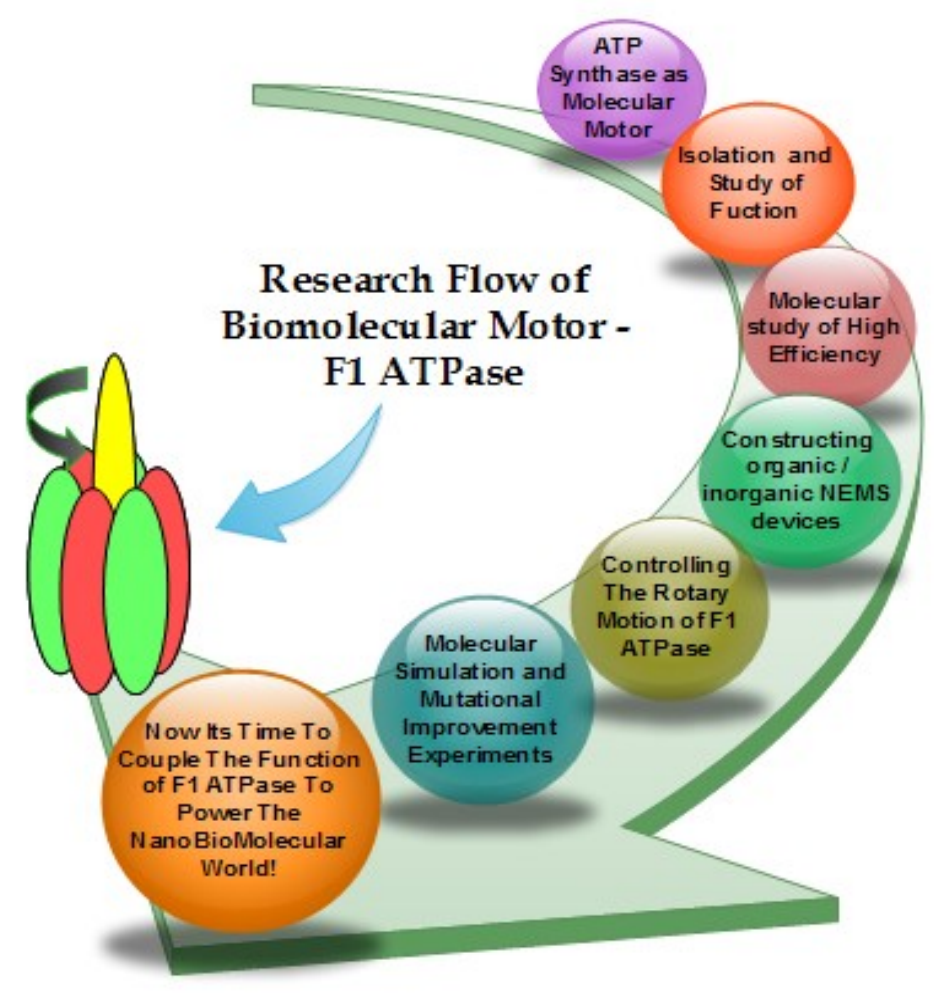

Fig. 1. Research exploration of F1 ATPase related study.

The primary goal of this brief review is to explain past though applications and explores novel possibilities for F1 ATPase as nanobioengine and describing possibilities to use F1 ATPase as nanobioengine in present or future microbiotechnological or nanobiotechnological applications.

Biomolecular Motors (Nanobiomachines). Scientists have been studying a wide range of biological nano-molecular motors (nanobiomachines), which includes number of motor proteins such as kinesin [5,6] dynein (Fig. 2a), RNA polymerase [7], myosin [8] (Fig. 2b), and adenosine triphosphate (ATP) synthase $[9,10,11,12,13]$ (Fig. 2c), function as nanoscale linear or rotary biological motors. 
(a)

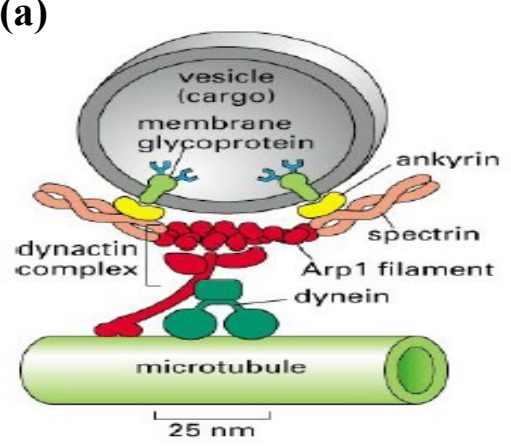

(b) Myosin II

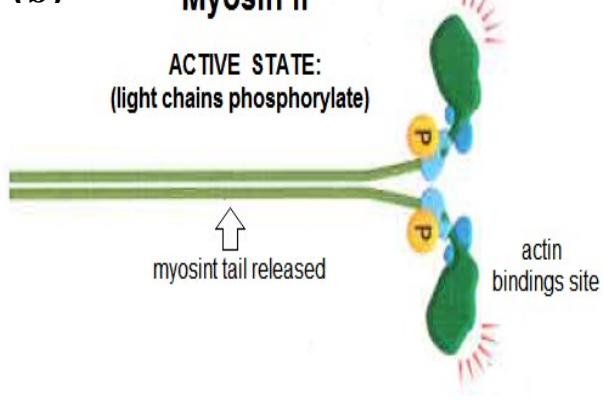

(c)

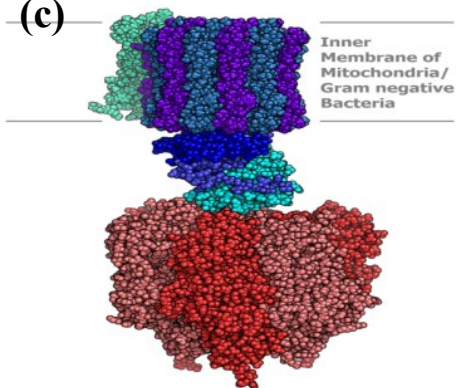

Fig. 2. Structures of (a) Dynein [14], (b) Myosin [15] and (c) The ATP synthase with subunits arrangement [16].

Different biological molecular motors are capable to generate or exert force by consuming energy currency of the cell (ATP). Fig. 3 compares some biomolecular motors for their functional characteristics (max. force generation and max efficiency). Myosin is the molecular motor with lowest energy conversion efficiency (20\%) with max force generation is $\sim 3 \mathrm{pN}$. Although RNA polymerase with same energy conversion efficiency (20\%) but exerts force of $\sim 14 \mathrm{pN}$. Kinesin functions with $50 \%$ energy conversion efficiency exert lesser force $(\sim 5 \mathrm{pN})$ than RNA polymerase [17]. F1 ATPase has highest energy conversion efficiency (100\%) with exerting force of 40pN [11]. 


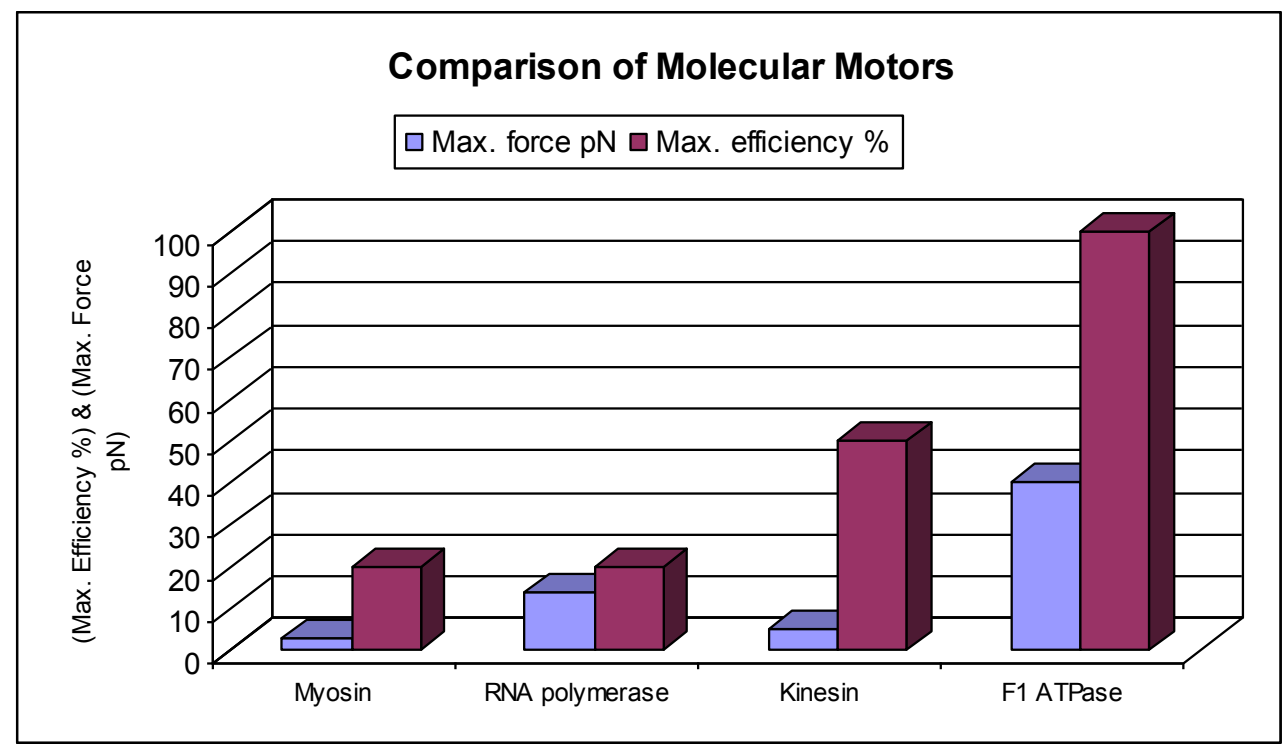

Fig. 3. Comparison of different molecular motors for maximum force generation and energy conversion efficiency $[11,17]$.

Bacterial flagellum (Fig. 4a) is one of the powerful moving biomolecular protein complex [18] which functions similar way like F0 Motor (Fig. 4b), rotates by using the electrochemical potential of hydrogen ions, without using ATP as fuel, to propel the bacterial cell. But this complex molecular structure creates a strong torque $(\sim 4000 \mathrm{pN})$ at stall and at its rim a force of $200 \mathrm{pN}$ governing high speed of rotation (400 to1700 rps) which can able to rotate flagellae of $10 \mu \mathrm{m}$ length with highly efficient way by using proton gradient or ionic electrochemical potential [19]. 

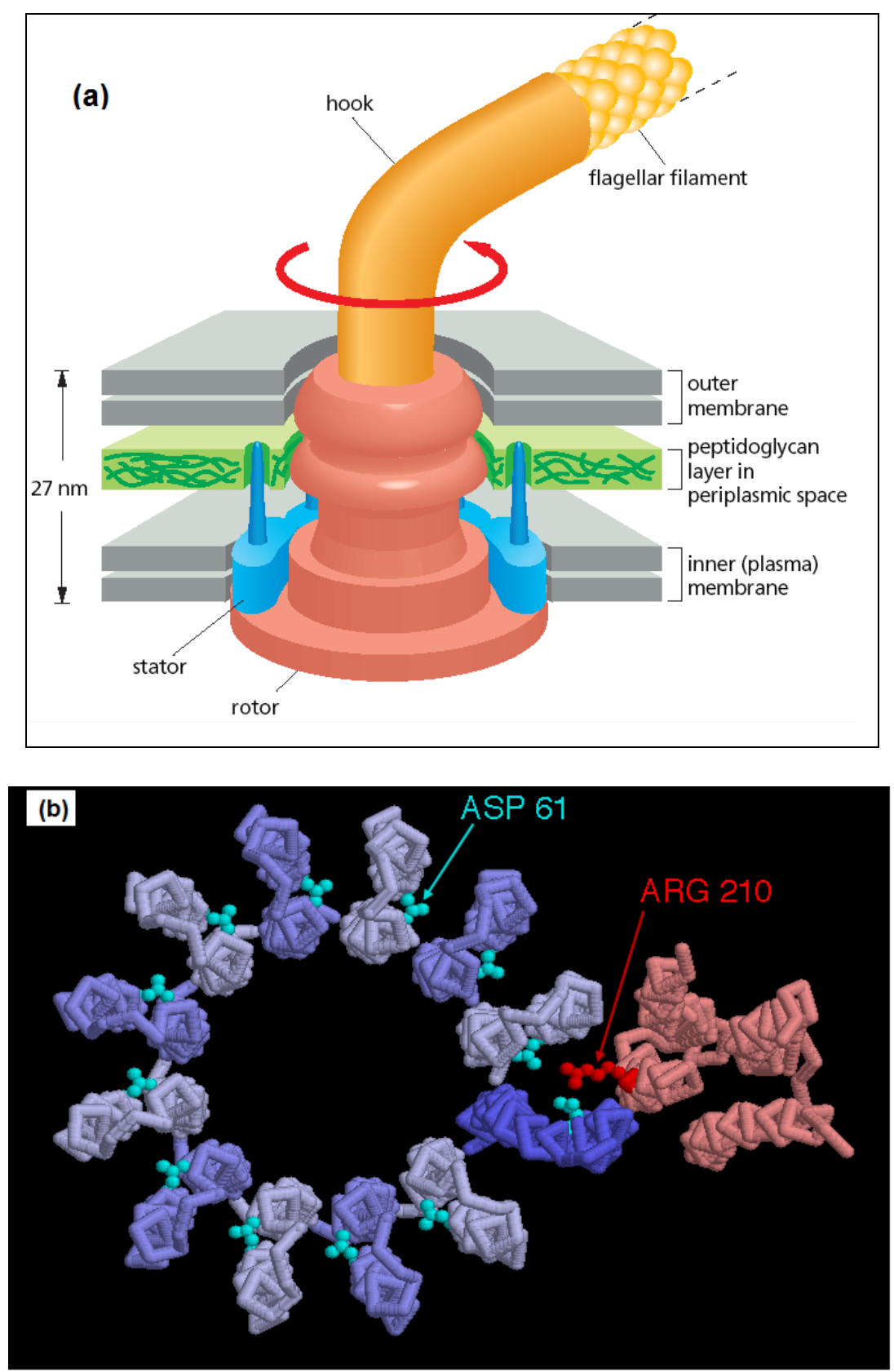

Fig. 4. (a) Bacterial flagellar assembly [18], (b) F0 motor of ATP synthase [21].

Electrochemical potential driven torque generation capacity by of bacterial flagella, bacterial retracting pilus and F0 subunit (c ring) of the ATP synthase were compared. Bacterial pilus retraction can be able to generate force $\sim 120 \mathrm{pN}$ by hydrolyzing ATP [20]. In case of Fo motor, it can generate the $45 \mathrm{pN}$ of torque required to turn F1 backwards and release newly synthesized ATP from the catalytic site [23]. Fig.4 shown the assembly and Fig. 5 compares the mechanical force 
exerted by molecular motors by using ionic electrochemical potential $[19,20,21,22,23]$, where bacterial flagellar motor generates maximum torque with high electrochemical potential conversion capacity to torque than bacterial retracting pilus and F0 ring of ATP synthase.

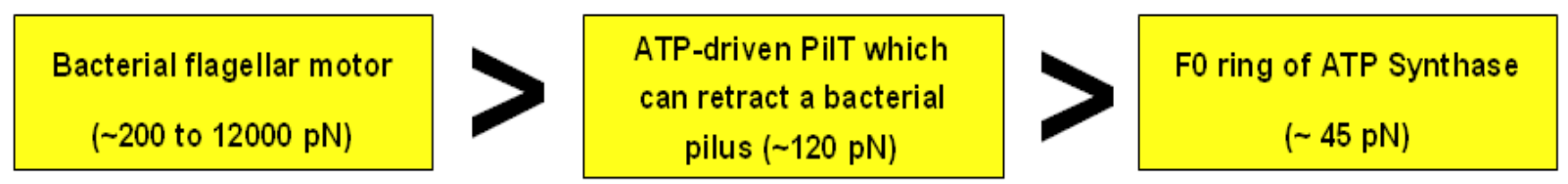

Fig. 5. Comparison of forces generation by biomolecular assemblies driven by proton gradient

Fuels for Nanobiomachines. Organism contains high energy organic molecules to drive life through series of reactions (oxidation/ reduction). These organic molecules include phosphoric anhydrides (ATP, ADP), an enol phosphate (PEP), an acyl phosphate (acetyl phosphate), and a guanidino phosphate (creatine phosphate), thioesters, such as acetyl-CoA which haves high free energy of hydrolysis. ATP structure [24] shown in Fig. 6 is exceptionally situated with the very high energy phosphates acts as a fuel in course of metabolic reactions. ATP biosynthesis takes place by many ways like substrate level phosphorylation, oxidative phosphorylation in cellular respiration and photo-phosphorylation in photo-synthesis by photo-synthetic organisms. ATP synthase makes ATP at rates over 100 molecules /second [25]. The average adult human, (weight of $70 \mathrm{~kg}$ ) consumes approximately 65 kilograms of ATP per day, an amount nearly equal to his/her own body weight! [26].

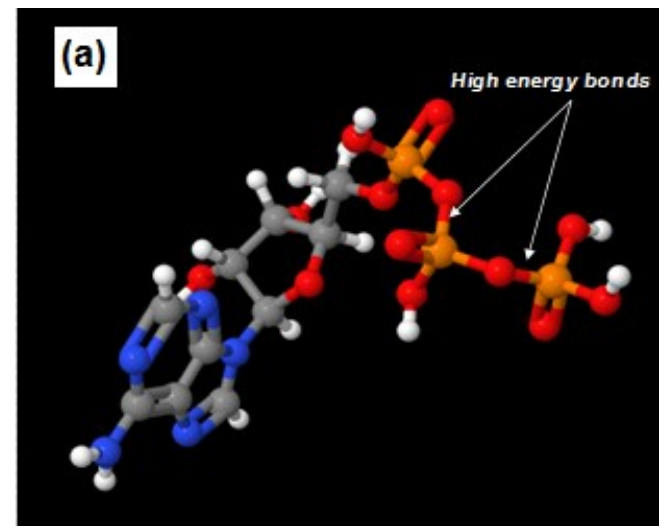

(b)

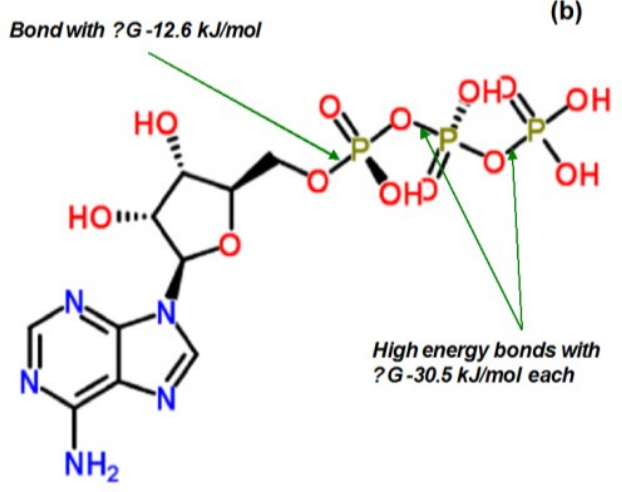

Fig. 6. High energy phosphate bonds shown in (a) 3D, (b) 2D views of an ATP molecule [24]. 
ATP Synthase as Nanobioengine. Adenosine triphosphate synthase (ATP synthase or F1F0 ATPase) is the world's smallest rotating motor showed in Fig. 1c, and it efficiently creates ATP (power or energy), hence the smallest power generator in cell's power houses (mitochondria, chloroplasts) and in bacteria. ATP synthase is the combination of two rotating motors (the F1 and F0) that are about $\sim 10 \mathrm{~nm}$ in diameter and height and are easily separated. It's a complex enzyme with distinct F1 and F0 part, embedded with biological membrane by F0 part. F1 contains 9 protein subunits $(3 \alpha+3 \beta+1 \gamma+1 \delta+1 \varepsilon)$ which can acts as ATP Synthase to produce ATP or as nanobioengine with rotary motion of the middle $\gamma$ shaft. F0 normally contains 15 protein subunits $(12 c+2 b+1 a)$ and c subunit acts as rotor when with membrane potential. ATP synthase displays a number of mechanisms for regulation of the enzyme's activity, such as the inhibition of ATP synthase by MgADP bound to the catalytic site [27].

\section{F1 ATPase Acts as Nanobioengine.}

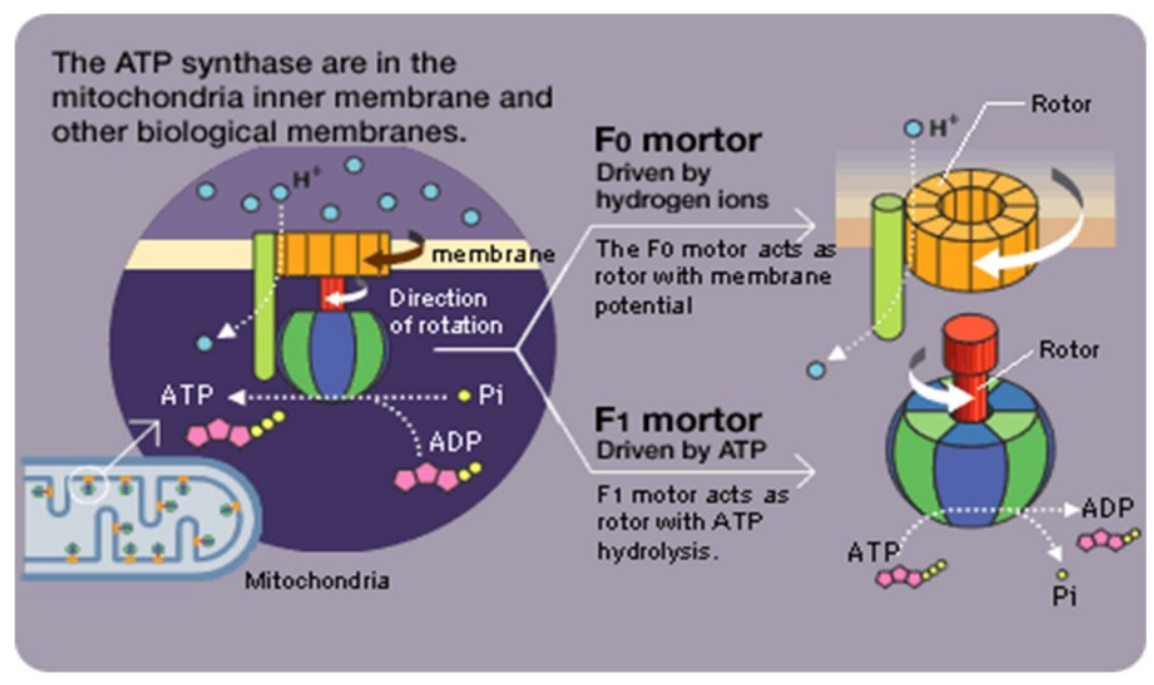

Fig. 7. F1 ATPase as biomolecular motor [28].

The F0 rotates by utilizing the hydrogen ion or proton concentration difference on the both sides of the biological membrane and responsible to rotate $\gamma$ shaft of ATP synthase to produce ATP from the ADP and phosphoric acid. F1 subunit synthesize ATP when $\gamma$ shaft rotates clockwise, but interestingly this enzyme subunit gives reversible rotation (anti-clockwise) of $\gamma$ shaft by consuming hydrolyzing ATP separately (Fig.7). Some bacteria use this reversed reaction to transport protons for 
maintaining the PMF under low oxygen conditions [27]. The F1-ATPase molecule, $8 \mathrm{~nm}$ in diameter and $14 \mathrm{~nm}$ in length, is capable of producing; 80 to $100 \mathrm{pN}$ of rotary torque [11,12]. It has been rotating within the organism for at least two billion years [29]. If we compare the function and molecule assembly of F1-ATPase with a mechanical engine (that generates electricity, pumps water, or compresses gas etc), similar nanoscale functions can be imagined for F1-ATPase by considering its working fashion as nano scale biological engine, so it's a nanobioengine of the cell.

Study of F1 ATPase. Junge et al. did experiment to observe rotation of $\gamma$ shaft (Fig. 8a). F1-ATPase was supported by His-tag engineered into the protein at the N-terminus to a glass surface with the beta-subunit. The counter-clockwise motion was detected under conditions of ATP-hydrolysis by attaching a fluorescent tagged actin filament to the $\gamma$-subunit [30] shown in Fig. 8a.

As Boyer [31] stated "ATP synthase, a splendid molecular machine" and the heart of this machine is F1 molecule. Extraordinary work made by a group of scientists in Kinosita's laboratory from Keio University, Japan, observed rotation of $\gamma$ shaft of a single F1 molecule microscopically. They have attached a long, thin, fluorescent actin polymer to $\gamma$ shaft and watched through CCD camera as ATP was hydrolyzed with uneven rotation in three discrete steps of $120^{\circ}$ by hydrolyzing 3 ATPs (One ATP per step). Some recent results suggest that $80^{\circ}$ rather than $90^{\circ}$ is closer to the actual substep size, the difference being an experimental error, or that the $80^{\circ}$ rotation is driven by ATP binding and the remaining $10^{\circ}$ by another process such as ATP hydrolysis [32].

The rotational and ATP hydrolysis rates are proportional to the ATP concentration in the submicromolar range. By calculation of frictional drag force on the long actin polymer and the rate of ATP hydrolysis, Kinosita's group concluded that the efficiency of this rotational mechanism in converting chemical energy into motion is nearly $100 \%$ [11]. In recent inspections results imply that the F1-ATPase achieves a nearly 100\% free energy conversion efficiency even far from quasistatic process for both the mechanical-to-chemical and chemical-to-mechanical transductions and such a 
high efficiency of a finite-time operation is not expected for macroscopic engines and highlights a remarkable property of the nanosized engines working in the energy scale of kBT [33].

Montemagno Research Group at Cornell University did detailed experimentation for the powering nanoelectromechanical device (NEMS) through energy derived from rotational torque produced by $\gamma$ shaft of F1 ATP Synthase $[9,10,13]$. They have studied unknown engineering properties of this enzyme by attaching a nanofabricated substrate ( 1 micrometer microsphere) to $\gamma$ shaft of F1-ATPase (Fig. 8b) and analyzed radial displacement of the microsphere and the angle of deformation of the gamma subunit. Attachment of microsphere and $\gamma$ shaft did by biotin- streptavidin linkage. Observation of microsphere movement was measured using a differential interferometer and moving images captured by CCD kinetics camera.
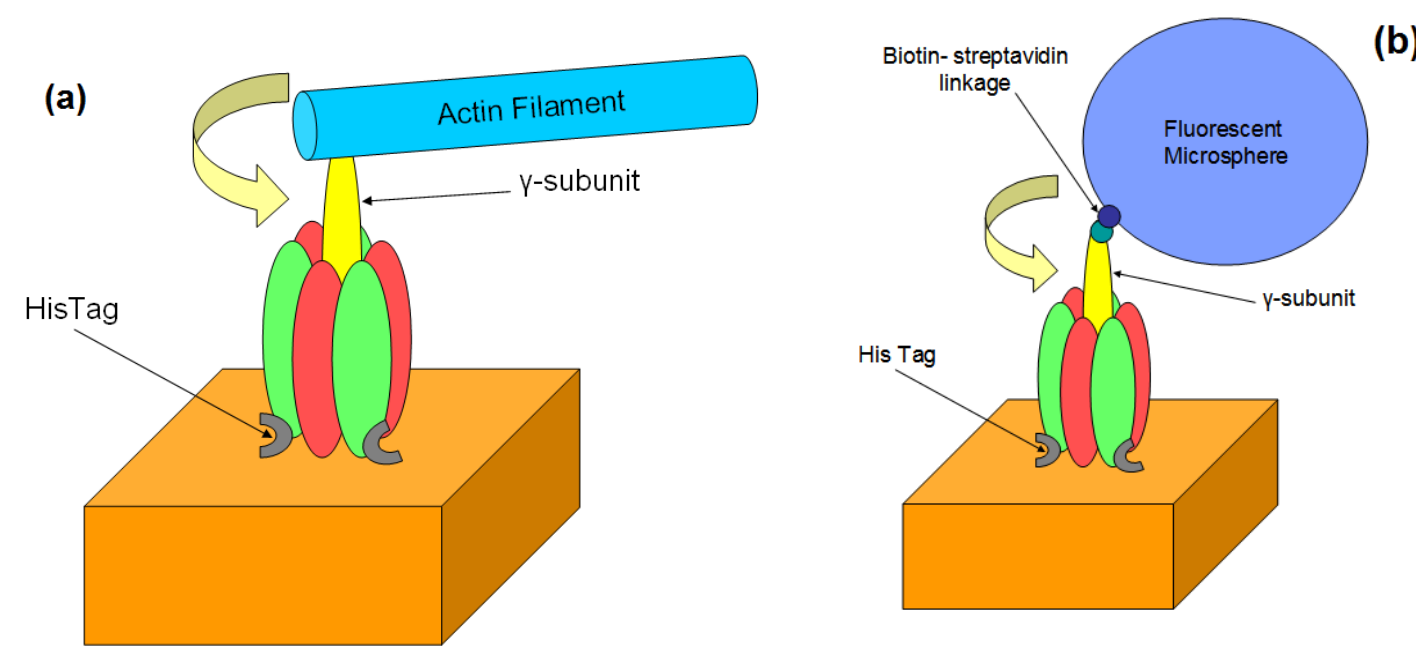

Fig. 8. (a) Counterclockwise rotation of actin filament [30], (b) Assembly F1 ATPase and fluorescent streptavidin coated nanofabricated substrate [34].

Detail molecular level efficiency evaluation of F1 ATPase to rotate with load were analyzed and suggested that the order of ATP hydrolysis, product release, and substrate binding may change as the result of external load on the F1 ATPase motor [35], this study giving detailed insight of substrate utilization and by product formation pattern which helps in designing fuel supply for F1 ATPase. Torque profiling of F1 ATPase in both ATP hydrolysis and synthesis direction were revels 
decoupling of ATP synthesis at lower torque [36], which explains high torque will produce by hydrolyzing ATP. Some recent findings on the cooperative interplay for torque generation between subunits of F1 ATPase explained by high-speed atomic force microscopy observation of counterclockwise cyclically propagation of three $\beta$ subunits giving insight on responsible torque generation factors [37]. Free energy simulations also explore the conformational change of the $\beta$ subunit proposing steps of torque generation in the F1 ATPase by characterizing two steps changing of the hydrogen-bond network around ATP and the dynamic movement of the C-terminal domain via sliding of the B-helix [38]. But still some aspects of the rotary mechanism of F1 ATPase while confessing us that, we are far from understanding [32].

Possible Future Applications of F1 ATPase as Nanobiomolecular Motor. An application of tomorrow's technology and developments obviously depends on believing today's imaginations. Number of nanobiomolecules haves tremendous scope to be used in nanotechnological applications in biomedical and physical science. Recently some applications described like membrane-associated motors for a gene or drug delivery at a constant ATP dependent velocity across a nanopore, a biosensor known as of Lab-on-a-Chip device able to detect changes on the distance of the DNA molecule [39]. Another approach of ATP synthesis by using light energy put on by Choi and Montemagno and they showed (Fig. 9) that the combining the functions of bacteriorhodopsin and F1-ATP-ase in a polymeric vesicle membrane production of ATP by using light energy [40]. 


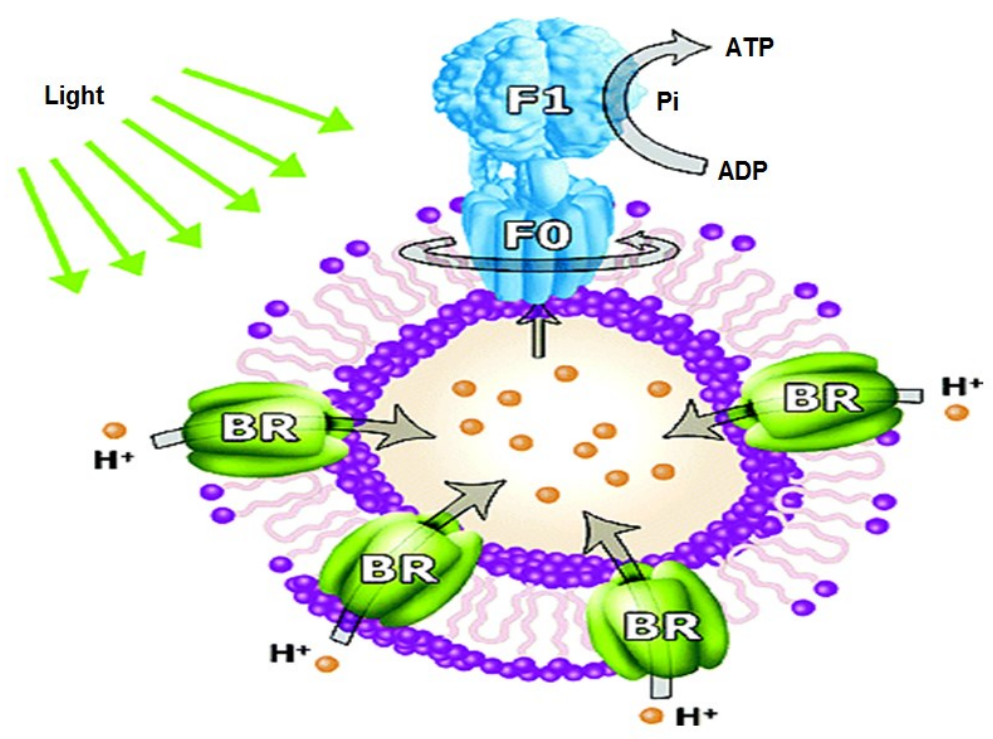

Figure 9. ATP synthesizing nanofactory [40].

As light driven bacteriorhodopsin pumps proton to create proton gradient inside the vesicle and by using that transmembrane gradient ATP synthase converts ADP into ATP and this research produced ATP synthesizing nanofactory. Furthermore we can imagine the F1 ATPase powering the operations of nanogate (Fig. 10a), also can be assembled for nanopumping system (Fig. 10b). Tremendous efforts of Montemagno's group from Cornell University towards powering organic and inorganic worlds by biological motors and chemical energy sources proposed future application explains the integration of biomolecular motor devices and cell-signalling systems and control of membrane implanted nanodevices by using the cell's sensory system [41]. 


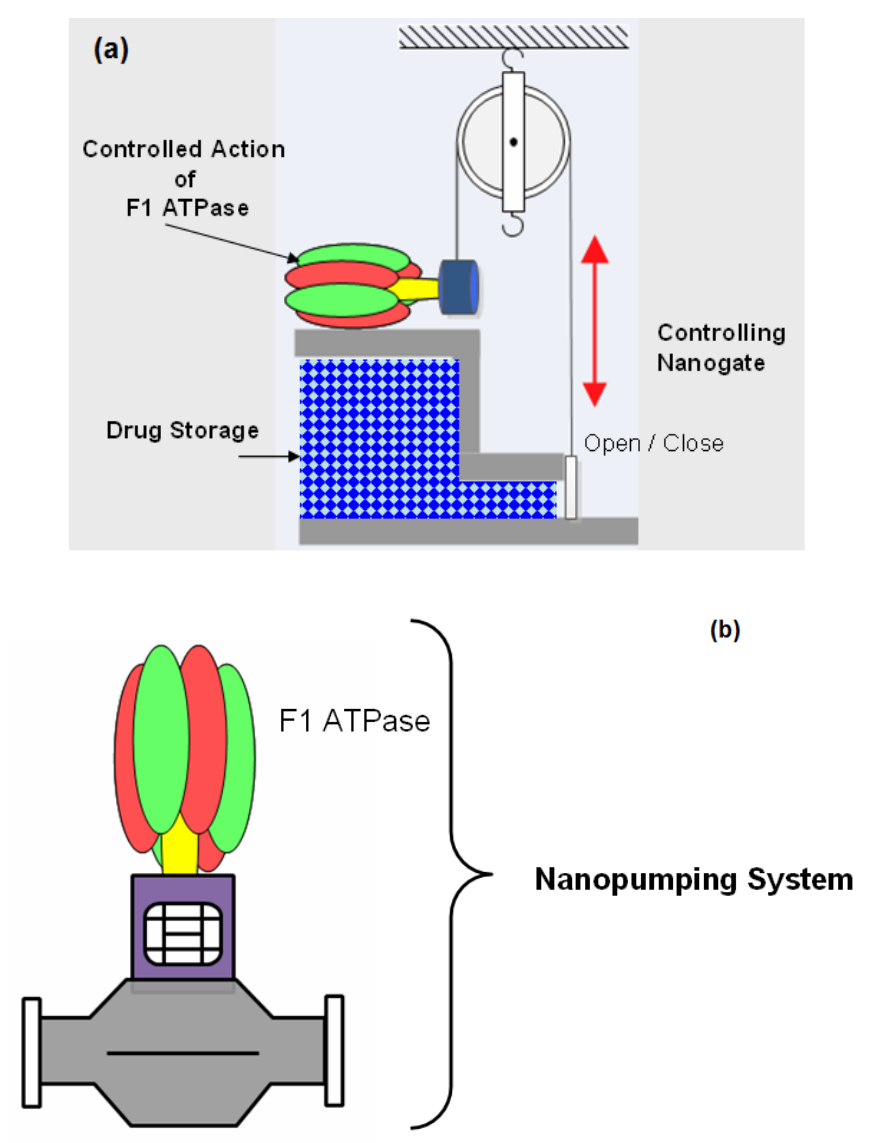

Fig. 10. (a) F1 ATPase powering and controlling operations of nanogate, (b) F1 ATPase powered nanaopumping system.

Another future application suggested by Montemagno Research Group to develop smart micro or nanopharmacies by using a rotation of F1 ATPase to deliver drugs to respective diseased cells (Fig. 11). Then next generation pharmaceuticals will be tiny pharmacies inside living cells powered by F1ATPase [42]. 


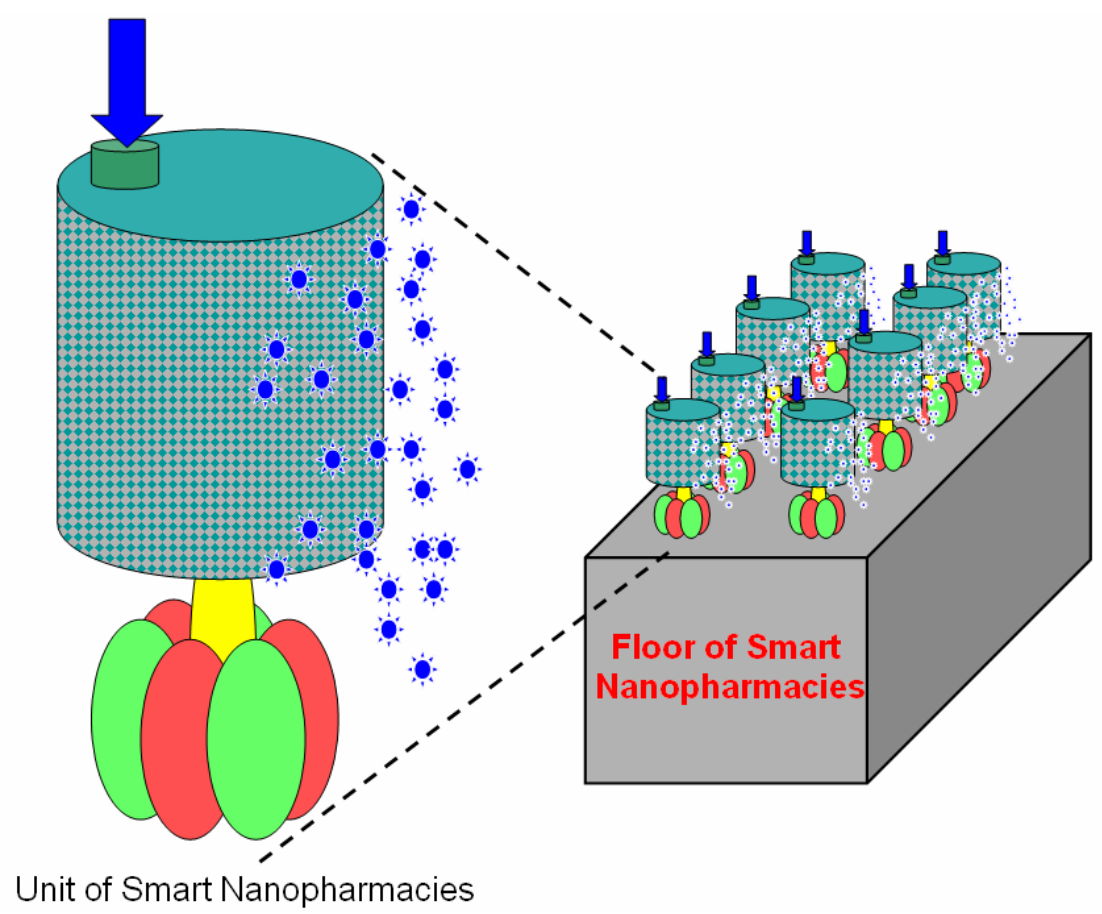

Fig. 11. Smart nanopharmacies to deliver drugs to respective diseased cells.

But there's still much insufficiency in nano-bio-robot and drug delivery therapy [43]. Recent advances in microelectromechanical systems (MEMS) have provided a unique opportunity to fabricate miniature biomedical devices for a variety of applications ranging from implantable drug delivery systems to lab-on-a-chip devices [44]. Nanobioengines can be used to power the joints of nanoelectromechanical devices (NEMS), MEMS or nano-bio-robot, which could be used to provide very local and accurate drug delivery.

In a recent study, a fluorescence probe F1300 labeled in inner chromatophores was used as a pH indicator for detecting proton flux driven by ATP synthesis in F0F1 - ATPase. In this application they used the function of F0F1-ATPase for the development of a nanobiosensor application to detect Clenbuterol, which is a nano-biosensor and it can be developed for detections of chemical molecules by a semi-quantitative assay [45]. He et al. engineered layer-by-layer assembled microcapsules have proved to be a suitable cellular mimetic structure, which can be applied for engineering active biomimetic systems with a cellular process and an added benefit is that these assembled microcapsules can be used as bioenergy containers and thus ATP supply on demand by using functional ATP synthase [46]. 
A concept described by Gilleo about micro-robots or "nanobots" and they will travel through the body to clear arteries (clear a blood clot) and make repairs, with suitable name macro-world submarine, the even smaller autonomous Bio-MEMS vehicle will be used in the future as internal medicine. Use of F1 ATPase in the concept of nano or micro level self-propelled submarine part as a propeller or movement related functions may find functional compatibility and helps to run these tiny machines without since they can get power from blood-borne nutrients [47].

Future Aspects and Conclusion. It seems very safe to predict that the more we learn about cells and organisms, the more intriguing will be the new mysteries that remain to be solved [48] and we expect more than those findings. So the study and applications of F1 ATPase, which are very different than F0 ATPase and flagellar motor, as providing ATP as fuel to get mechanical rotational energy will be in a more controlled way to handle functions of nano mechanical output. Other two motors (F0 ATPase and flagellar motor) are functioning to generate force derived from proton motive force across the membrane, which will be not easy to use those bioengines for their functional application. Further ATP synthase should be studied to harness the energy produced by $\gamma$ shaft for the various applications (Fig. 12) like, (i)To propel the nano or micro devices local, controlled and accurate drug delivery; (ii) Rotating motor in nanopumping systems with controlled operation; (iii) Functions with nanogears for opening and closing nanogates or nanovalves; (iv) As fluid propeller to cool or lowering the temperature of nanodevices functioning in bioenvironment; (v) Functioning as nanobiodriller to open or clean blocked blood vesicles. 


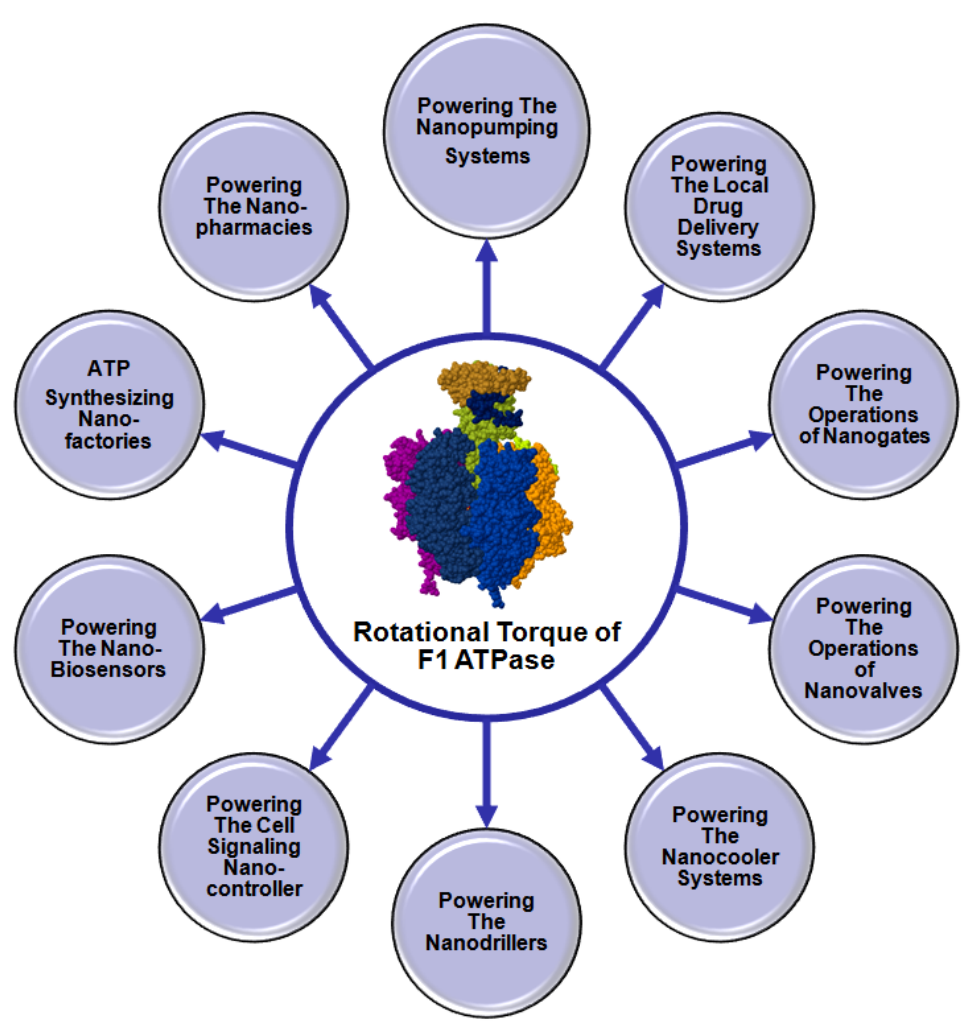

Fig. 12. Futuristic nano applications possibly powered by F1 ATPase

Among the all biomolecular motors, F1 ATPase (part of ATP synthase) may deliver the expectations of high speed rotary motion with unique characteristic of extreme energy conversion efficiency. One day F1 ATPase will be major revolutionary part of future nanobiotechnology or nanotechnology, where it can be in charge as nanoscale motor, which will be responsible for powering the nano motions and nano actions. 


\section{References}

[1] S. Abeer, Future Medicine: Nanomedicine, JIMSA 25 (2012)187-192.

[2] S. Furuike, M.D. Hossain, Y. Maki, K. Adachi, T. Suzuki, A. Kohori, H. Itoh, M. Yoshida, K. Kinosita, Axle-less F1-ATPase rotates in the correct direction, Science 319 (2008) 955958.

[3] A. Lohrasebi, Y. Jamali, H. Rafii-Tabar, Modeling the effect of external electric field and current on the stochastic dynamics of ATPase nano-biomolecular motors, Physica A 387 (2008) 5466-5476.

[4] S. Yamaguchi, S. Matsumoto, K. Ishizuka, Y. Iko, K.V. Tabata, H.F. Arata, H. Fujita, H. Noji, I. Hamachi, Thermally Responsive Supramolecular Nanomeshes for On/Off Switching of the Rotary Motion of F1 - ATPase at the Single-Molecule Level, Chemistry-A European Journal 14 (2008) 1891-1896.

[5] S.M. Block, Kinesin: What Gives? Minireview, Cell 93 (1998) 5-8.

[6] M.J. Schnitzer, S.M.Block, Kinesin hydrolyses one ATP per 8-nm step, Nature 388 (1997) 386-390.

[7] M.D. Wang, M.J. Schnitzer, H. Yin, R. Landick, J. Gelles, S.M. Block, Force and velocity measured for single molecules of RNA polymerase, Science 282 (1998) 902-907.

[8] K. Kitamura, M. Tokunaga, A.H. Iwane, T. Yanagida, A single myosin head moves along an actin filament with regular steps of 5.3 nanometres, Nature 397 (1999) 129-134.

[9] C. Montemagno, G. Bachand, S. Stelick, M. Bachand, Constructing biological motor powered nanomechanical devices, Nanotechnology 10 (1999) 225-231.

[10] G. Bachand, C. Montemagno, Constructing organic/inorganic NEMS devices powered by biomolecular motors, Biomedical Microdevices 2 (2000) 179-184. 
[11] R. Yasuda, H. Noji, K. Kinosita, M. Yoshida, F1-ATPase Is a Highly Efficient Molecular Motor that Rotates with Discrete 120 Steps, Cell 93 (1998) 1117-1124.

[12] H. Noji, R. Yasuda, M. Yoshida, K. Kinosita, Direct observation of the rotation of F1ATPase, Nature 386 (1997) 299-302.

[13] R. Soong, G. Bachand, H. Neves, A. Olkhovets, H. Craighead, C. Montemagno, Powering an inorganic nanodevice with a biomolecular motor, Science 290 (2000) 1555-1558.

[14] A.A. Johnson, J. Lewis, M. Raff, K. Roberts, P. Walter, The cytoskeleton, in: M. Anderson, S. Granum (Eds.), Molecular Biology of the Cell, 5th edition, Garland science, Taylor \& Francis Group, New York, 2007 pp.1022.

[15] A.A. Johnson, J. Lewis, M. Raff, K. Roberts, P. Walter, The cytoskeleton, in: M. Anderson, S. Granum (Eds.), Molecular Biology of the Cell, 5th edition, Garland science, Taylor \& Francis Group, New York, 2007 pp.1025.

[16] Adapted from the Wikimedia Commons file "Image: Atp Synthase.png" [http://en.wikipedia.org/wiki/File:Atp_synthase.PNG]

[17] Y.M. Romanovsky, A.N. Tikhonov, Molecular energy transducers of the living cell. Proton ATP synthase: a rotating molecular motor, Physics-Uspekhi 53 (2010) 893.

[18] A.A. Johnson, J. Lewis, M. Raff, K. Roberts, P. Walter, Mechanisms of Cell Communication, in: M. Anderson, S. Granum (Eds.), Molecular Biology of the Cell, 5th edition, Garland science, Taylor \& Francis Group, New York, 2007 pp.943.

[19] G. Oster, H. Wang, Rotary protein motors, Trends in cell biology, 13 (2003) 114-121.

[20] D. Kaiser, Bacterial motility: How do pili pull? Current Biology 10 (2000) R777-R780.

[21] Image from the RCSB PDB (www.pdb.org) of PDB ID 1 C17 (V.K. Rastogi, M.E. Girvin, Structural changes linked to proton translocation by subunit c of the ATP synthase, Nature,402 (1999) 263-268.

[22] B. Maier, L. Potter, M. So, H. Seifert, M.P. Sheetz, Single pilus motor forces exceed 100 pN, PNAS 99 (2002) 16012-16017. 
[23] G. Oster, H. Wang, Reverse engineering a protein: the mechanochemistry of ATP synthase. BBA 1458(2) (2000) 482-510.

[24] CSID:5742 on [http://www.chemspider.com/Chemical-Structure.5742.html]

[25] A.E. Senior, ATP synthase: Motoring to the finish line, Cell 130 (2007) 220-221.

[26] R. Garrett, C.M. Grisham, Biochemistry, Belmont, CA: Brooks/Cole, Cengage Learning 2010.

[27] Bald, ATP Synthase: Structure, Function and Regulation of a Complex Machine, in: G.A. Peschek et al. (Eds.), Bioenergetic Processes of Cyanobacteria, Springer, Netherlands, 2011, pp. 239-261.

[28] Reprinted (adapted) with permission from "H. Noji, Research of rotating molecule, ATP synthetase, Biohistory journal, Winter (2004).” Copyright 2004 JT Biohistory Research Hall. [http://www.brh.co.jp/en/seimeishi/journal/043/research_11.html]

[29] H. Noji, Research of rotating molecule, ATP synthetase, Biohistory Journal, Winter (2004). Website: http://www.brh.co.jp/en/seimeishi/journal/043/research_11.html

[30] W. Junge, H. Lill, S. Engelbrecht, ATP synthase: an electrochemical transducer with rotatory mechanics, Trends Biochem. Sci. 22 (1997) 420.

[31] P.D. Boyer, The ATP synthase-a splendid molecular machine, Annu. Rev. Biochem. 66 (1997) 717-749.

[32] K. Kinosita Jr., K. Adachi, H. Itoh, Rotation of F1-ATPase: How an ATP-driven molecular machine may work, Annu. Rev. Biophys. Biomol. Struct. 33 (2004) 245-268.

[33] S. Toyabe, E. Muneyuki, Nanosized free-energy transducer F1-ATPase achieves 100\% efficiency at finite time operation, arXiv preprint arXiv: 1210.4017 (2012).

[34] G.D. Bachand, R.K. Soong, H.P. Neves, A, Olkhovets, H.G. Craighead, C.D. Montemagno, Precision attachment of individual F1-ATPase biomolecular motors on nanofabricated substrates. Nano Lett. 1 (2001) 42-44. 
[35] D. Spetzler, R. Ishmukhametov, T. Hornung, L.J. Day, J. Martin, W.D. Frasch, Single molecule measurements of F1-ATPase reveal an interdependence between the power stroke and the dwell duration, Biochemistry 48 (2009) 7979-7985.

[36] A. Palanisami, T. Okamoto, Torque-induced slip of the rotary motor F1-ATPase, Nano Lett. 10 (2010) 4146-4149.

[37] T. Uchihashi, R. Iino, T. Ando, H. Noji, High-speed atomic force microscopy reveals rotary catalysis of rotorless F1-ATPase, Science 333 (2011) 755-758.

[38] Y. Ito, T. Oroguchi, M. Ikeguchi, Mechanism of the conformational change of the F1ATPase $\beta$ subunit revealed by free energy simulations, J. Am. Chem. Soc. 133 (2011) 33723380.

[39] E. Cabezon, V.F. Lanza, I. Arechaga, Membrane-associated nanomotors for macromolecular transport, Curr. Opin. Biotechnol. 23 (2012) 537-544.

[40] Reprinted (adapted) with permission from "H. Choi, C. Montemagno, Artificial organelle: ATP synthesis from cellular mimetic polymersomes. Nano Lett. 5 (2006) 2538-2542.

[41] R. Segelken, Fantastic voyage: Tiny pharmacies propelled through the body could result from Cornell breakthrough in molecular motors, 1999 on [http://www.news.cornell.edu/releases/sept99/bio_nano_mechanical.hrs.html]

[42] R. Segelken, How Cornell scientists uncover and remake molecules of life. On [http://www.news.cornell.edu/chronicle/00/10.12.00/ss-molecules_of_life.html]

[43] Liu, S. Wang, W. Xu, Q. Liang, Bio-nano-robot and drug targeting delivery technology, Chinese Journal of Mechanical Engineering 44 (2008) 80-86.

[44] C. Kaparissides, S. Alexandridou, K. Kotti, S. Chaitidou, Recent advances in novel drug delivery systems, Journal of Nanotechnology Online 2 (2006) 1-11.

[45] C. Cunshe, L. Xiaojuan, Application of a biosensor for super sensitive detector of clenbuterol, N.Z. J. Agric. Res. 50 (2007) 689-695. 
[46] Q. He, L. Duan, W. Qi, K. Wang, Y. Cui, X. Yan, J. Li, Microcapsules containing a biomolecular motor for ATP biosynthesis, Adv. Mater. 20 (2008) 2933-2937.

[47] K. Gilleo, The Sci-Fi Future of Medicine ... the Next 50 Years. On [www.allflexinc.com/PDF/Future\%20of\%20Medicine.pdf]

[48] B. Alberts, Cell Biology: The Endless Frontier, Molecular Biology of the Cell 21(2010) $3785-3785$. 